\title{
Damping mechanisms of phonon polaritons, exploited by stimulated Raman gain measurements
}

\author{
U. T. Schwarz* and Max Maier \\ Naturwissenschaftliche Fakultät II - Physik, Universität Regensburg, D-93040 Regensburg, Germany
}

(Received 14 October 1997; revised manuscript received 26 January 1998)

\begin{abstract}
Using a two-beam amplifier experiment stimulated Raman gain measurements were performed for the polariton branch of the $256-\mathrm{cm}^{-1} A_{1}$ (TO) optical phonon mode in $\mathrm{ZnO}$-doped congruent $\mathrm{LiNbO}_{3}$ and undoped nearly stoichiometric $\mathrm{LiNbO}_{3}$ for polariton frequencies from 30 to $230 \mathrm{~cm}^{-1}$. The dependence of the peak value and linewidth of the Raman gain curve on the polariton frequency provides detailed information on the frequency-dependent damping of the polariton. Spontaneous Raman spectra of the $256 \mathrm{~cm}^{-1}$ phonon, with particular emphasis upon the low-frequency wing of the Raman line, complete the results. The experimental results are carefully analyzed by comparison with a theory connecting the stimulated and spontaneous Raman data to the dielectric function of $\mathrm{LiNbO}_{3}$, which contains the damping of the polaritons. As damping mechanisms the anharmonic decay of the phonon part of the polariton, the scattering at crystal defects and the coupling to low-frequency excitations are discussed. We relate three of the low-frequency excitations to $E$ phonon modes of $\mathrm{LiNbO}_{3}$ and the major part to defect modes. [S0163-1829(98)05925-6]
\end{abstract}

\section{INTRODUCTION}

The dispersion relation and damping of phonon polaritons in crystals has been the subject of many investigations in recent years. The experimental methods used in these studies include impulsive stimulated Raman scattering and transient grating experiments with femtosecond pulses, ${ }^{1-7}$ nonlocal time-delayed coherent anti-Stokes Raman scattering (CARS) with picosecond pulses, ${ }^{8-10}$ amplifier measurements of the gain factor of stimulated Raman scattering (SRS) from polaritons with nanosecond pulses, ${ }^{11}$ and cw spontaneous Raman scattering from polaritons. ${ }^{12-14}$ The benefits and drawbacks of these methods can be seen from the discussion of the results for the ferroelectric crystal lithium niobate, which is of particular interest in nonlinear optics and electro-optics.

The coupling of the polaritons to low-frequency excitations has been investigated in congruent $\mathrm{LiNbO}_{3}$ using transient grating experiments with femtosecond pulses. ${ }^{6}$ A splitting of the polariton dispersion curve due to the mode coupling was observed. However, no specific information on the polariton damping in $\mathrm{LiNbO}_{3}$ was obtained, in contrast to $\mathrm{LiTaO}_{3}$ where the frequency dependence of the damping was determined. ${ }^{7,15}$ The frequency range of this method is limited from about $30 \mathrm{~cm}^{-1}$ to the linewidth of the femtosecond pulses of about $150 \mathrm{~cm}^{-1}$. In $\mathrm{LiNbO}_{3}$ measurements below room temperature are difficult with this method, because the high intensity of the femtosecond laser pulses causes photorefractive damage. ${ }^{7}$

The propagation of phonon polaritons of the $256-\mathrm{cm}^{-1} A_{1}$ mode in $\mathrm{MgO}$-doped and congruent $\mathrm{LiNbO}_{3}$ has been studied using a nonlocal time-delayed CARS technique. ${ }^{10}$ The frequency dependence of the polariton damping was measured in the range from 10 to $80 \mathrm{~cm}^{-1}$ at 77 and $300 \mathrm{~K}$. The frequency range is limited by the fact that the propagation of the polaritons can be followed only over distances from the $\mathrm{mm}$ to the $\mathrm{cm}$ range. The damping of the polaritons in $\mathrm{LiNbO}_{3}$ in this frequency range is determined by the coupling to low-frequency modes at about $120 \mathrm{~cm}^{-1}$ and to a
Debye relaxational mode, which is important at $300 \mathrm{~K}$, but can be neglected at $77 \mathrm{~K}$.

In principle, stimulated and spontaneous Raman scattering from polaritons ${ }^{16}$ provide the same information. The information on the damping of the polaritons is contained in the Raman linewidth, which can be obtained from the spontaneous Raman spectra and the stimulated Raman gain curve. The advantage of the Raman gain measurements is the high spectral resolution, which is mainly determined by the narrow width of the laser lines, over the complete range of polariton frequencies. The spectral resolution of spontaneous Raman scattering from polaritons is low in the lowfrequency, linear part of the dispersion curve, because it is determined by the group velocity of the polaritons and the finite solid angle of the detected scattered light. A spectral resolution of about $20 \mathrm{~cm}^{-1}$ has been obtained for example for $\mathrm{LiTaO}_{3}$ in the frequency range from 50 to $160 \mathrm{~cm}^{-1} .13$ This resolution is typical for spontaneous Raman scattering from polaritons, but it is not sufficient to extract information on the damping of the polaritons. The spectral resolution for stimulated scattering, where a laser linewidth and a corresponding resolution of much less than $1 \mathrm{~cm}^{-1}$ are possible without problems, is much better. In the frequency range close to the optical-phonon frequency the spectral resolution of both methods is comparable, but spontaneous Raman scattering has the advantage of being the simpler method with a better signal-to-noise ratio.

Inspite of the high spectral resolution of Raman gain measurements, this method has not been used for the investigation of polariton damping until recently. Room-temperature measurements ${ }^{11}$ of the gain factor of stimulated Raman scattering from polaritons using a two-beam amplifier setup provided information on the polariton damping in $\mathrm{MgO}$-doped $\mathrm{LiNbO}_{3}$ in the frequency range from 80 to $230 \mathrm{~cm}^{-1}$. Different damping mechanisms of the polariton in $\mathrm{LiNbO}_{3}$ have been discussed. Below $80 \mathrm{~cm}^{-1}$ information on the damping of polaritons is obscured by the effect of propagation of the polaritons out of the excitation region. Above $230 \mathrm{~cm}^{-1}$ the gain is too small to be detectable, because the large phase- 
matching angle between the pump and Stokes beam decreases the interaction length.

In this paper the damping mechanisms of phonon polaritons of the $256-\mathrm{cm}^{-1} A_{1}$ mode of $\mathrm{LiNbO}_{3}$ are carefully studied and analyzed using both stimulated and spontaneous Raman scattering from polaritons. The measurements were carried out at liquid-nitrogen temperature in doped congruent $\mathrm{LiNbO}_{3}$ and undoped nearly stoichiometric $\mathrm{LiNbO}_{3}$. It is the combination of both Raman methods and the comparison of doped and nearly stoichiometric crystals which provide new information on the number and type of low-frequency excitations determining the polariton damping over almost the complete frequency range. A description of the Raman amplifier experiments and theory, based on classical coupled wave equations, is given in Secs. II and III, respectively. The influence of the propagation of the polaritons out of the excitation volume on the gain factor and the damping mechanisms of the polaritons, anharmonic decay, scattering at defects, and coupling to low-frequency excitations, are presented in Sec. IV. The physical origin of the lowfrequency excitations is discussed in Sec. V, followed by the conclusions (Sec. VI).

\section{STIMULATED RAMAN AMPLIFIER EXPERIMENT}

In the SRS amplifier experiment a pump laser beam and a second laser beam at the Stokes frequency are radiated into the crystal. The stimulated amplification of the Stokes beam is measured. The frequency $\omega$ of the excited polariton is given by the difference of the fixed pump laser frequency $\omega_{P}$ and the tunable Stokes frequency $\omega_{S}$ :

$$
\omega=\omega_{P}-\omega_{S} .
$$

The wave vector $\mathbf{k}$ of the polariton is fixed by noncollinear phase-matching with phase-matching angle $\vartheta$ shown in the lower part of Fig. 1:

$$
\mathbf{k}=\mathbf{k}_{P}-\mathbf{k}_{S} .
$$

In contrast to generator or oscillator experiments, both the frequency $\omega$ and wave vector $\mathbf{k}$ of the polariton can be varied independently. This means that in the amplifier experiment polaritons can be excited, whose frequency and wave vector do not fulfill the dispersion relation. Polaritons lying on the dispersion curve are characterized by the frequency $\omega_{\Pi}$ and the wave vector $\mathbf{k}_{\Pi}$. The scattering geometry is shown in Fig. 1. All wave vectors lie in the isotropic $x-y$ plane of the crystal with polarizations parallel to the optical axis $\hat{c}$ to excite polaritons of $A_{1}$ symmetry. Because of the low divergence of the Stokes and pump beams, the wave vector of the polariton is defined with high accuracy.

For the SRS amplifier experiment two time-synchronized laser pulses with a frequency difference tunable from zero to several hundred wave numbers are required. The pump beam with a wavelength of $532 \mathrm{~nm}$ was provided by a frequencydoubled single-frequency Nd:YAG laser, delivering pulses with a duration of $\tau_{1 / e}=13.5 \mathrm{~ns}$ and an energy of $60 \mathrm{~mJ}$. A dye laser, which was pumped with the third harmonic of the Nd:YAG laser, was used as source for the Stokes beam. The

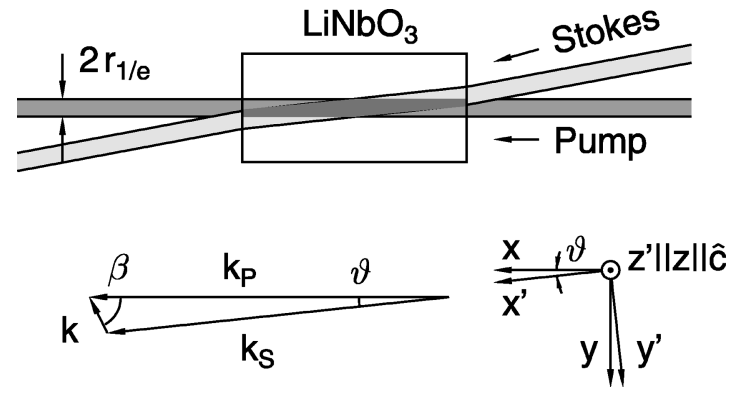

FIG. 1. Scattering geometry for the Raman amplifier experiment and noncollinear phase-matching triangle. The pump beam is incident perpendicular to the crystal surface and propagates in the $x$ direction. The Stokes beam propagates in the $x^{\prime}$ direction. All polarizations are parallel to the $z$ direction, which is identical with the optical $\hat{c}$ axis of the uniaxial lithium niobate crystal. $\vartheta$ is the phasematching angle of the pump and Stokes beam inside the crystal.

laser dye coumarin 153 spanned the wavelength range $\lambda_{S}$ $=520-565 \mathrm{~nm}$. The duration of the Stokes pulses was $6 \mathrm{~ns}$ and the energy was $0.1 \mathrm{~mJ}$.

Each beam was focused by a separate lens system to the same beam radius of typically $r_{1 / e}=0.7 \mathrm{~mm}$. Because the confocal parameter of about $2 \mathrm{~m}$ was large compared to the crystal length $l=25 \mathrm{~mm}$, the pump and Stokes beam radii in the crystal were approximately constant. Pump pulse, incident Stokes pulse, and amplified Stokes pulse were detected with one vacuum photodiode (Hamamatsu S20) using different optical delay lines, and recorded on a transient digitizer (time resolution of the detection system $0.5 \mathrm{~ns}$ ). A detailed description of the experimental setup is given in Ref. 17.

The power amplification $A=P_{S a} / P_{S i}$ was determined from the amplified and incident Stokes powers, $P_{S a}$ and $P_{S i}$, at the time of the maximum of the pump pulse. ${ }^{18} \mathrm{We}$ measured the amplification $A$ for a certain polariton wave number $\bar{\nu}_{\Pi}=\omega_{\Pi} /(2 \pi c)$ on the dispersion curve at the corresponding phase-matching angle $\vartheta$. Then we tuned the Stokes wave number $\bar{\nu}_{S}$, while keeping the phase-matching angle $\vartheta$ constant. In this way the wave number $\bar{\nu}$ of the excited polariton is varied [see Eq. (1)]. The corresponding change of the polariton wave vector $\mathbf{k}$ which follows from Eq. (2) is small and can be neglected in the vicinity of the polariton branch. Therefore the amplification $A$ is measured as a function of $\bar{\nu}$ for nearly constant $\mathbf{k}$.

In $\mathrm{LiNbO}_{3}$ the photorefractive effect and the dark traces due to the high pulse energy of the nanosecond pump pulse caused most of the difficulties in the amplifier experiment. The corresponding optical damage decreased in the sequence congruent, stoichiometric, $\mathrm{MgO}$-doped and $\mathrm{ZnO}$-doped $\mathrm{LiNbO}_{3}$. In order to reduce the problems with optical damage, we measured at low pump intensities of $I_{P 0}=10$ to $100 \mathrm{MW} / \mathrm{cm}^{2}$, corresponding to an amplification of $A=1.5$ to 20 . We further used a mechanical shutter to select single laser pulses from the $10 \mathrm{~Hz}$ pulse train, to avoid accumulation of the optical damage. After the measurements the photorefractive effect and the dark traces were healed by heating the crystal to room temperature for $2 \mathrm{~h}$ and in the case of stoichiometric $\mathrm{LiNbO}_{3}$ by irradiation with incoherent light from a focused $100 \mathrm{~W}$ halogen lamp. We investigated $\mathrm{ZnO}$ doped and $\mathrm{MgO}$-doped congruent $\mathrm{LiNbO}_{3}$ (crystals dimensions $\left.x \times y \times z=25 \times 8 \times 10 \mathrm{~mm}^{3}\right)$ and undoped nearly sto- 
ichiometric $\mathrm{LiNbO}_{3} \quad(\mathrm{Li}: \mathrm{Nb}=49.5: 50.5, \quad x \times y \times z=10 \times 9$ $\times 11 \mathrm{~mm}^{3}$ ) at $T=77 \mathrm{~K}$. Because of the large photorefractive effect it was not possible to record gain curves in undoped congruent $\mathrm{LiNbO}_{3}$.

For a comparison with theory we have to evaluate the gain factor $G_{S}$ from the experimental data. In the plane-wave approximation the incident Stokes intensity $I_{S i}$ is amplified exponentially with the gain factor $G_{S}$, which depends on the pump intensity $I_{P}$. The amplified Stokes intensity $I_{S a}$ is obtained by integration over the crystal length $l$ taking into account the experimental geometry. We get

$$
I_{S a}\left(y^{\prime}, z^{\prime}\right)=I_{S i}\left(y^{\prime}, z^{\prime}\right) \exp \left\{\int_{-l / 2}^{l / 2} G_{S}\left(I_{P}\left(x^{\prime}, y^{\prime}, z^{\prime}\right)\right) d x^{\prime}\right\} .
$$

The $x^{\prime}, y^{\prime}$, and $z^{\prime}$ dependence of $G_{S}$ is determined by the radial pump intensity distribution $I_{P}(y, z)$, with $y$ $=x^{\prime} \sin \vartheta+y^{\prime} \cos \vartheta$ and $z=z^{\prime}$ (see Fig. 1). The spatial profile of the pump and Stokes beams was close to Gaussian, as was veryfied with a CCD camera. Knowing the intensity distributions of the pump beam and the incident Stokes beam, we can integrate Eq. (3) over the Stokes beam profile in the $y^{\prime}-z^{\prime}$ plane to obtain the amplification of the Stokes power $A=P_{S a} / P_{S i}$. All integrations were done numerically to get the gain factor $G_{S}$ as a function of the measured amplification $A$ for a fixed geometry and of the pump intensity $I_{P 0}$ in the center of the beam, ${ }^{17}$ which is determined experimentally.

The measurements were carried out for a large number of polariton frequencies $\bar{\nu}_{\Pi}$ and the corresponding phasematching angles $\vartheta$. The measured gain curves $G_{S}$ for

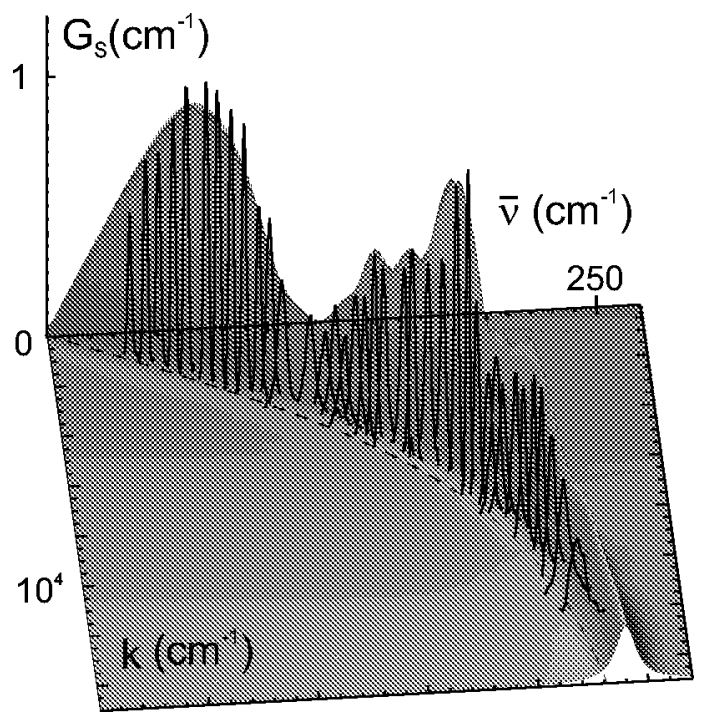

FIG. 2. Gain factor $G_{S}$ as a function of the polariton frequency $\bar{\nu}$ and wave vector $k$ in $\mathrm{ZnO}: \mathrm{LiNbO}_{3}$ at $T=77 \mathrm{~K}$. Each solid gain curve represents a measurement for a polariton frequency $\bar{\nu}_{\Pi}$ and the corresponding phase-matching angle $\vartheta$. The shaded surface is calculated using Eq. (6) and including the damping mechanisms of Sec. IV. Dashed curve: polariton dispersion curve.

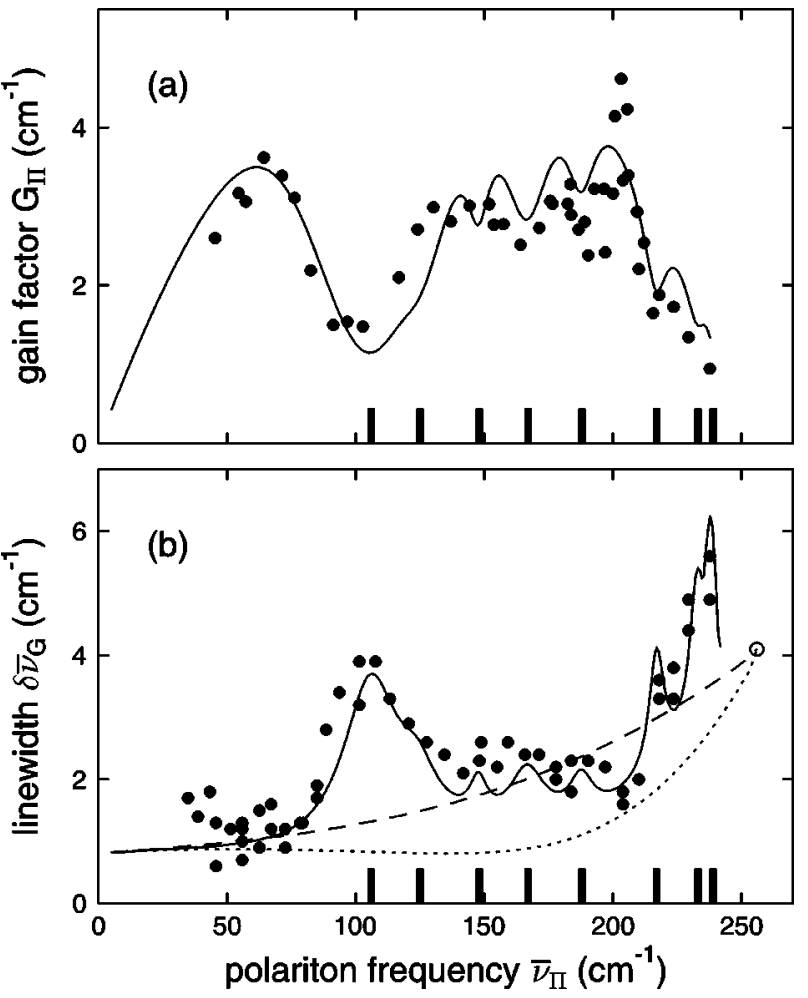

FIG. 3. (a) Gain factor $G_{\Pi}$ and (b) linewidth $\delta \bar{\nu}_{G}$ versus polariton frequency $\bar{\nu}_{\Pi}$ in nearly stoichiometric $\mathrm{LiNbO}_{3}$ at $T=77 \mathrm{~K}$. Full circles: experimental points; solid curve: simulation including all damping mechanisms; dashed curve: constant damping $\Gamma_{0}$; dotted curve: frequency-dependent damping $\Gamma_{\Pi}$ without low-frequency modes. Short vertical lines: resonance frequencies of low-frequency modes.

$\mathrm{ZnO}: \mathrm{LiNbO}_{3}$ at $T=77 \mathrm{~K}$ and $I_{P 0}=80 \mathrm{MW} / \mathrm{cm}^{2}$ are plotted over the $\bar{\nu}-k$ plane (solid curves in Fig. 2) and evolve as a ridge over this plane. It should be noted that the ridge shows several peaks that are not expected from previous calculations ${ }^{16,19,20}$ based on a single polar phonon mode model. Therefore, we calculated the Raman gain $G_{S}$ using Eq. (6) of Sec. III taking into account the additional damping mechanisms discussed in Sec. IV [Eqs. (8), (10), (11), and (13)]. The result is shown in Fig. 2 as the gray-shaded surface.

Although Fig. 2 gives a good overview of the gain factor in the $\bar{\nu}-k$ plane, it is not suitable for a quantitative comparison between theory and experiments. We determined therefore the height $G_{\Pi}$ of the maximum and the width $\delta \bar{\nu}_{G}$ of the measured and calculated gain curves for different polariton frequencies $\bar{\nu}_{\Pi}$. The results are shown for nearly stoichiometric $\mathrm{LiNbO}_{3}$ and $\mathrm{ZnO}$-doped $\mathrm{LiNbO}_{3}$ at $77 \mathrm{~K}$ in Figs. 3 and 4 , respectively. The symbols represent the experimental points and the curves correspond to the calculations, which will be discussed in detail in Sec. IV. From reference measurements in benzene ${ }^{17}$ we estimate the error for the determination of $\bar{\nu}_{\Pi}$ and $\delta \bar{\nu}_{G}$ to about $20 \%$ of the linewidth. The accuracy for the frequency dependence of the gain factor $G_{\Pi}$ is also about 20\%. The most striking features in Figs. 3 and 4 are the maxima and minima of $G_{\Pi}$ and $\delta \bar{\nu}_{G}$, which are related to the coupling of the polaritons to low-frequency modes. The frequencies of these modes are marked in the figures by short vertical lines. At these frequencies the damp- 

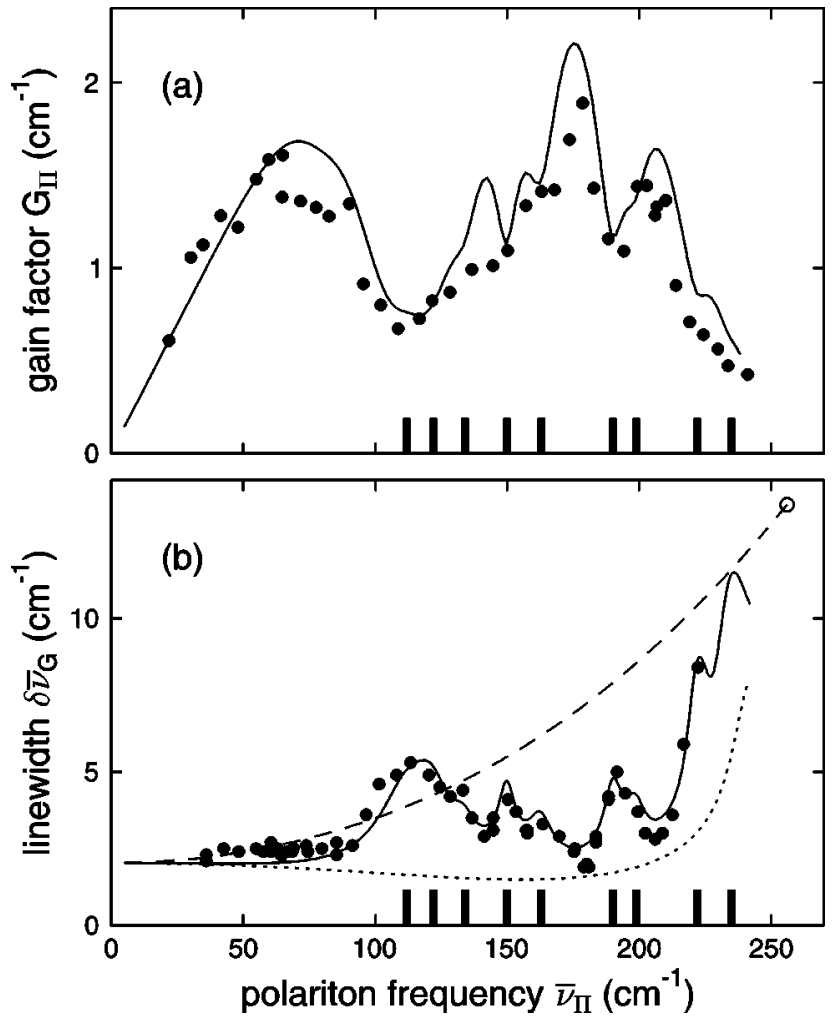

FIG. 4. (a) Gain factor $G_{\Pi}$ and (b) linewidth $\delta \bar{\nu}_{G}$ in $\mathrm{ZnO}: \mathrm{LiNbO}_{3}$ at $T=77 \mathrm{~K}$. Symbols as in Fig. 3.

ing of the polaritons is high, leading to maxima of linewidth $\delta \bar{\nu}_{G}$ and the corresponding minima of the gain factor $G_{\Pi}$.

\section{PLANE-WAVE THEORY OF STIMULATED RAMAN GAIN}

For the theoretical description we follow the ansatz of nonlinear coupled plane waves of Henry and Garrett ${ }^{19}$ and of Sussman. ${ }^{20}$ The evolution of the Stokes field and the electromagnetic field of the polariton are described by wave equations, while for the mechanical part of the polariton the usual damped oscillator equation is used. For the small Stokes gain in our experiment, the depletion of the pump field $E_{P}$ can be neglected. Using the slowly varying amplitude approxima- tion and eliminating the vibrational coordinate of the polariton, the following coupled differential equations for the amplitudes of the Stokes and polariton electromagnetic fields, $E_{S}$ and $E_{\Pi}$, are obtained:

$$
\begin{aligned}
\frac{\partial E_{S}}{\partial x}= & \frac{i}{2 k_{S}}\left\{-\left(k_{S}^{2}-\frac{\omega_{S}^{2}}{c^{2}} \varepsilon_{S}\right) E_{S}+\frac{\omega_{S}^{2}}{c^{2}}\left[\left(d_{33}+d_{\mathrm{Q}} \chi_{Q}^{*}\right)\right.\right. \\
& \left.\left.\times E_{P} E_{\Pi}^{*}+d_{\mathrm{Q}}^{2} \chi_{Q}^{*}\left|E_{P}\right|^{2} E_{S}\right]\right\}, \\
\frac{\partial E_{\Pi}}{\partial x}= & \frac{i}{2 k \cos \beta}\left\{-\left(k^{2}-\frac{\omega^{2}}{c^{2}} \varepsilon_{\Pi}\right) E_{\Pi}\right. \\
& \left.+\frac{\omega^{2}}{c^{2}}\left(d_{33}+d_{\mathrm{Q}} \chi_{Q}\right) E_{P} E_{S}^{*}\right\} .
\end{aligned}
$$

$k_{S}, \omega_{S}$, and $\varepsilon_{S}$ are the wave vector, frequency, and dielectric constant of the Stokes light. $\beta$ is the angle between the pump beam and the polariton beam (see Fig. 1). The nonlinear coefficients $d_{33}$ and $d_{Q}$ take into account the electronic and vibrational contributions to the amplification process.

The essential quantity in Eqs. (4a) and (4b) is the dielectric function $\varepsilon_{\Pi}$, which is given by

$$
\varepsilon_{\Pi}=\varepsilon_{\infty}+\frac{S_{0} \omega_{0}^{2}}{\omega_{0}^{2}-\omega^{2}-i \Gamma_{0} \omega}=\varepsilon_{\infty}+\chi_{Q} .
$$

Since we are interested only in the lowest polariton branch, the higher branches are taken into account by the effective dielectric constant $\varepsilon_{\infty} . S_{0}$ is the oscillator strength and $\Gamma_{0}$ the damping constant of the optical phonon. We have introduced the abbreviation $\chi_{Q}$ for the frequency-dependent part of the dielectric function $\varepsilon_{\Pi}$.

We insert an exponential amplification of the electromagnetic fields with a gain factor $\frac{1}{2} G_{S}$ into Eqs. (4a) and (4b) and get a quadratic equation for the gain factor $G_{S}$, from which $G_{S}$ is calculated as a function of polariton frequency $\omega$ and wave vector $k . \mathrm{In} \mathrm{LiNbO}_{3}$ for polariton frequencies from 30 to $230 \mathrm{~cm}^{-1}$ the following simplifications are a good approximation. The second term in the square brackets of Eq. (4a) and the $G_{S}^{2}$ term in the quadratic equation for $G_{S}$ are neglected. Then, we get the following result for the gain factor:

$$
G_{S}(\omega, k)=\frac{2 \omega_{S} I_{P}}{c^{2} \varepsilon_{0} \eta_{S} \eta_{P}} \operatorname{Im}\left\{\frac{\left(\varepsilon_{\Pi}-\varepsilon_{\infty}\right)\left[d_{Q}^{2}\left(k^{2} c^{2} / \omega^{2}-\varepsilon_{\infty}\right)+2 d_{33} d_{Q}\right]+d_{33}^{2}}{k^{2} c^{2} / \omega^{2}-\varepsilon_{\Pi}}\right\} .
$$

$\varepsilon_{0}$ is the dielectric constant of the vacuum, $\eta_{S}$ and $\eta_{P}$ are the refractive indices at the Stokes and pump frequencies, respectively. In the above approximation the gain factor $G_{S}$ for polariton scattering is proportional to the pump intensity $I_{P}$. It can be shown that the gain factor $G_{S}$ of Eq. (6) is equivalent to the gain factor derived by Barker and Loudon ${ }^{16}$ using a response function theory of Raman scattering from polaritons. $G_{S}$ is proportional to the differential spontaneous scattering cross section $d^{2} \sigma / d \Omega d \omega$ of Ref. 16, indicating that the linewidth $\delta \bar{\nu}_{G}$ measured in our amplifier experiments is equal to the linewidth in spontaneous scattering, as is well known for Raman scattering from molecular vibrations and phonons. $^{21}$

In the numerical calculations values of the nonlinear coefficients are needed. The nonlinear coefficient $d_{33}$ is nearly frequency independent in the infrared spectral range. We take the value ${ }^{22} d_{33}=-34.4 \mathrm{pm} / \mathrm{V}$ measured for frequency doubling at $\lambda=1064 \mathrm{~nm}$. The coupling constant 
$d_{Q}$ is calculated from the Raman-scattering cross section ${ }^{23}$ to be $d_{\mathrm{Q}}=-18 \mathrm{pm} / \mathrm{V} \cdot{ }^{17}$ The sign of $d_{\mathrm{Q}}$ was chosen to be the same as that of $d_{33}$ (see Ref. 20).

\section{DAMPING MECHANISMS OF THE POLARITON}

In the preceding section a single polar phonon mode model with frequency-independent damping constant $\Gamma_{0}$ has been used for the description of the polaritons and the calculation of the polariton gain factor. It has been shown, ${ }^{6,7,11}$ however, that this simple model cannot describe correctly the dispersion relation and damping of the polaritons. We discuss in the following sections additional damping mechanisms of the polariton, which have been identified in $\mathrm{LiNbO}_{3}$.

\section{A. Polaritons leaving the interaction volume}

In $\mathrm{LiNbO}_{3}$ the group velocity $\mathrm{v}_{\mathrm{gr}}=\partial \omega_{\Pi} / \partial k_{\Pi}$, which corresponds to the slope of the dispersion curve, is in good approximation constant for polaritons with frequencies less than $100 \mathrm{~cm}^{-1}$. As the polaritons in this frequency region travel with the high velocity $\mathrm{v}_{\mathrm{gr}} \approx c / 5$ and have a lifetime exceeding several tens of picoseconds, they can travel macroscopic distances of several millimeters to centimeters in the crystal. This has been observed experimentally in $\mathrm{LiNbO}_{3}$ by use of a nonlocal time-delayed CARS technique. ${ }^{10}$ The propagation of the polaritons acts as an effective damping mechanism in the SRS amplifier experiment, because polaritons, which have left the interaction volume of pump and Stokes beam, do not contribute to the amplification process.

We use a simple model, where the loss caused by the propagation of the polaritons out of the excitation volume is taken into account by introducing an additional effective absorption coefficient $\alpha_{\mathrm{pr}}$. Since the pump and Stokes beams have Gaussian intensity distributions, it is difficult to define the excitation volume precisely. We assume that the effect of polariton propagation over a distance $a r_{1 / e}$ is equivalent to a decrease of the polariton intensity by a factor of $e$. Here $a$ is a parameter, which determines the ratio of the effective diameter of the interaction region to the radius $r_{1 / e}$ of the laser beams. Since the polaritons propagate under an angle $\beta$ with respect to the laser beam (see Fig. 1), we define the effective absorption coefficient as

$$
\alpha_{\mathrm{pr}}=\frac{\sin \beta}{a r_{1 / e}} .
$$

The propagation loss is taken into account in the differential equation for the polariton field by adding the term $\alpha_{\mathrm{pr}} E_{\Pi}$ in the curly brackets on the right-hand side of Eq. (4b). When Eqs. (4a) and (4b) are solved in the way described above, it is found that the additional loss modifies the imaginary part of the dielectric constant in the following way:

$$
\operatorname{Im}\left(\varepsilon_{p r}\right)=\operatorname{Im}\left(\varepsilon_{\Pi}\right)-\frac{\operatorname{Re}\left(\varepsilon_{\Pi}\right)}{\omega} \frac{\mathrm{v}_{\mathrm{gr}} \sin \beta}{a r_{1 / e}} .
$$

With this modified dielectric function, the gain factor $G_{\Pi}$ and linewidth $\delta \bar{\nu}_{G}$ can be calculated using Eq. (6). In the range of low polariton frequencies $\left(<50 \mathrm{~cm}^{-1}\right.$ in $\left.\mathrm{LiNbO}_{3}\right)$, where the propagation loss dominates, this leads to a gain factor $G_{\Pi}$ proportional to the polariton frequency $\omega_{\Pi}$ and a constant linewidth $\delta \bar{\nu}_{G}$ in agreement with the experimental results (see Fig. 4). For higher frequencies other damping mechanisms become predominant and the group velocity decreases, therefore the contribution caused by propagation can be neglected.

We have checked experimentally the dependence of the linewidth $\delta \bar{\nu}_{G}$ on the radius $r_{1 / e}$ of the light beams in the range from 0.4 to $1.3 \mathrm{~mm}$ and found good agreement with the calculations using Eq. (8) (for $a=0.2$ ).

\section{B. Anharmonic decay into two acoustic phonons}

Information on the damping of optical phonons in $\mathrm{LiNbO}_{3}$ has been obtained from the investigation of the temperature dependence of the corresponding spontaneous Raman linewidths. ${ }^{24}$ The main mechanisms of phonon damping are the decay into two acoustic phonons and the scattering by thermal phonons and at crystal defects. These effects were found to be important in doped and undoped congruent $\mathrm{LiNbO}_{3}$. In stoichiometric $\mathrm{LiNbO}_{3}$ the scattering process can be neglected for the spontaneous Raman linewidth of the 256- $\mathrm{cm}^{-1} A_{1}$ mode. We assume that the damping mechanisms of the optical phonon are also effective for the damping of the phonon part of the polariton. But in contrast to the optical phonon, the polariton frequency is tunable. Therefore, the frequency dependence of the damping mechanisms has to be considered.

We discuss in this section the decay of the phonon part of the polariton into two acoustic phonons with frequencies $\omega_{\mathrm{ac}}^{\prime}$ and $\omega_{\mathrm{ac}}^{\prime \prime}$. Scattering of polaritons at thermal phonons can be neglected at $77 \mathrm{~K}$. Applying a theoretical model which has been used for TO and LA phonons to polaritons, the damping constant $\Gamma_{\text {de }}$ can be written as ${ }^{25}$

$$
\begin{aligned}
\Gamma_{\mathrm{de}}(\omega, \mathrm{T}) \propto & \omega^{-1} \sum_{\mathbf{k}_{\mathrm{ac}}^{\prime} \mathbf{k}_{\mathrm{ac}}^{\prime \prime}} \sum_{j_{\mathrm{ac}}^{\prime} j_{\mathrm{ac}}^{\prime \prime}} \frac{\left|\Phi\left(\mathbf{k} ; \mathbf{k}_{\mathrm{ac}}^{\prime}, j_{\mathrm{ac}}^{\prime} ; \mathbf{k}_{\mathrm{ac}}^{\prime \prime}, j_{\mathrm{ac}}^{\prime \prime}\right)\right|^{2}}{\omega_{\mathrm{ac}}^{\prime}\left(\mathbf{k}_{\mathrm{ac}}^{\prime}, j_{\mathrm{ac}}^{\prime}\right) \omega_{\mathrm{ac}}^{\prime \prime}\left(\mathbf{k}_{\mathrm{ac}}^{\prime \prime}, j_{\mathrm{ac}}^{\prime \prime}\right)} \\
& \times \Delta\left(\mathbf{k}-\mathbf{k}_{\mathrm{ac}}^{\prime}-\mathbf{k}_{\mathrm{ac}}^{\prime \prime}\right) \delta\left(\omega-\omega_{\mathrm{ac}}^{\prime}-\omega_{\mathrm{ac}}^{\prime \prime}\right) \\
& \times\left(n\left(\omega_{\mathrm{ac}}^{\prime}\right)+n\left(\omega_{\mathrm{ac}}^{\prime \prime}\right)+1\right) .
\end{aligned}
$$

$\mathbf{k}_{\mathrm{ac}}, j_{\mathrm{ac}}$, and $\omega_{\mathrm{ac}}\left(\mathbf{k}_{\mathrm{ac}}, j_{\mathrm{ac}}\right)$ represent the wave vector, branch, and frequency of the acoustic phonons involved in the decay process. $\Phi$ is the Fourier transform of the cubic anharmonic force constant and $\Delta$ states that the wave vectors must be conserved up to a reciprocal-lattice vector. $n(\omega, \mathrm{T})$ is the phonon occupation number in thermal equilibrium.

For simplicity, we neglect the anisotropy of the anharmonic interactions. We assume that the polariton decays into two acoustic phonons with the same frequency $\omega_{\mathrm{ac}}^{\prime}=\omega_{\mathrm{ac}}^{\prime \prime}$ $=\frac{1}{2} \omega$ in the dispersionless part of the acoustic phonon branches. It can be seen from the phonon-dispersion curves ${ }^{26}$ that this is a good approximation for $\mathrm{LiNbO}_{3}$. In this case the two-phonon density of states [sum over the second line in Eq. (9)] is proportional to $\omega^{2}$.

The decay of the polariton into two acoustic phonons is most important in the frequency region close to the optical phonon frequency (about $200-256 \mathrm{~cm}^{-1}$ in $\mathrm{LiNbO}_{3}$ ), because here the fraction of the mechanical energy of the po- 
lariton is largest. In this frequency region the polariton frequency is nearly independent of the wave vector, i.e., the polariton is similar to an optical phonon. The dependence of the Fourier transform $\Phi$ of the anharmonic force constant on the wave vector $k$ of the polariton is negligible, because the lattice motion corresponds to that of an optical phonon and the long polariton wavelength causes little anharmonicity. In the frame of the continuum theory of the acoustic phonons the Fourier transform $\Phi$ is proportional to the wave vector $k_{\mathrm{ac}}$ and therefore to the frequency $\omega_{\mathrm{ac}}$. For the symmetric decay into two acoustic phonons with half of the polariton frequency $\omega$, we arrive at $\Phi \propto \omega^{2}$. In summary, the damping constant is obtained from Eq. (9) to be

$$
\Gamma_{\text {de }}(\omega, \mathrm{T})=\Gamma_{0}\left(\omega / \omega_{0}\right)^{3}\left[2 n\left(\frac{1}{2} \omega, \mathrm{T}\right)+1\right] .
$$

It has been shown ${ }^{24}$ that in the limit $\omega=\omega_{0}$ this equation describes the temperature dependence of the spontaneous Raman linewidth of the $256-\mathrm{cm}^{-1} A_{1}$ mode in stoichiometric $\mathrm{LiNbO}_{3}$, when the anharmonic side lines are taken into account. The constant $\Gamma_{0}$ has been determined in this way to be $3.4 \mathrm{~cm}^{-1}$. We use this value also for doped and congruent $\mathrm{LiNbO}_{3}$ crystals. $^{24}$

We have calculated the linewidth $\delta \bar{\nu}_{G}$ in nearly stoichiometric $\mathrm{LiNbO}_{3}$ at $77 \mathrm{~K}$ taking into account the propagation of the polaritons out of the interaction region and the anharmonic decay [Eqs. (8) and (10), respectively]. The result is shown as dotted curve in Fig. 3(b), which decreases more strongly than the dashed curve which has been calculated using a constant value $\Gamma_{0}$ of the damping constant [in Eq. (5)]. The comparison with the experimental data shows that the calculated dotted curve provides the background for the maxima and minima of the experimental points in Fig. 3(b). The detailed structure of the experiments can only be reproduced when the coupling of the polaritons to low-frequency excitations (Sec. IV D) is taken into account.

For $\mathrm{ZnO}$-doped $\mathrm{LiNbO}_{3}$ [Fig. 4(b)] the decrease of the linewidth $\delta \bar{\nu}_{G}$ close to the optical phonon frequency is steeper than that of nearly stoichiometric $\mathrm{LiNbO}_{3}$ [Fig. 3(b)]. In a recent paper ${ }^{27}$ the influence of disorder in the crystal and of the concentration of defects on the anharmonic decay of phonons has been treated. With increasing defect concentration a stronger dependence of the damping constant on the phonon frequency than for the perfect crystal was predicted. Another mechanism causing a steeper decrease of the linewidth below the optical phonon frequency will be discussed in the next section.

\section{Scattering at imperfections}

The spontaneous Raman linewidth of the $256-\mathrm{cm}^{-1} A_{1}$ mode in $\mathrm{LiNbO}_{3}$ depends markedly on the crystal composition. ${ }^{24,28,29}$ It increases from stoichiometric $\mathrm{LiNbO}_{3}$ over congruent $\mathrm{LiNbO}_{3}$ to $\mathrm{ZnO}-$ and $\mathrm{MgO}$-doped $\mathrm{LiNbO}_{3}$. The differences between the linewidths of the nonstoichiometric and the stoichiometric crystals are temperature independent and have been attributed to elastic scattering of the phonons at crystal imperfections. ${ }^{24}$ Under the assumption of a frequency dependence of the scattering rate $\propto \bar{\nu}^{4}$, similar to Rayleigh scattering, the room-temperature measurements of the SRS gain factor $G_{\Pi}$ and linewidth $\delta \bar{\nu}_{G}$ have been explained. ${ }^{11}$ But for the low-temperature measurements of
SRS in doped $\mathrm{LiNbO}_{3}$ discussed in this paper the decrease of the linewidth $\delta \bar{\nu}_{G}$ in the frequency range below the TO phonon $\left(256 \mathrm{~cm}^{-1}\right)$ is much steeper than predicted by the $\bar{\nu}^{4}$ dependence. Therefore we have to discuss the scattering mechanism in more detail. We restrict our treatment to elastic scattering within the polariton branch, corresponding to a phase shift or change of the propagation direction of the polaritons, because there is not sufficient information available on the phonon branches of $\mathrm{LiNbO}_{3}$ to discuss other scattering processes.

As the polaritons are assumed to be scattered at defects, that are mainly $\mathrm{Nb}, \mathrm{Mg}$, or $\mathrm{Zn}$ on $\mathrm{Li}$ sites and $\mathrm{Nb}$ vacancies, ${ }^{30}$ we use a first-order perturbation theory based on the scattering of phonons due to mass differences. ${ }^{31}$ Different binding strengths have been treated in a similar way as different masses. When only one polariton branch is considered, the scattering rate is given by ${ }^{31}$

$$
\Gamma_{\mathrm{sc}}(\omega)=C \frac{k^{2} \omega^{2}}{\mathrm{v}_{\mathrm{gr}}(\omega)},
$$

where $C$ contains the volume of the unit cell and parameters characterizing the defects, e.g., the difference between the defect mass and the average mass in the crystal.

When the frequency $\omega$ of the polariton approaches the optical phonon frequency $\omega_{0}$, the group velocity $v_{\text {gr }}$ of the polaritons goes to zero and the perturbation theory breaks down. Therefore Eq. (11) should not be applied to calculate the spontaneous Raman linewidth of the optical phonon. In the low-frequency range $\left(<100 \mathrm{~cm}^{-1}\right)$, where $v_{g r}$ is frequency independent, the scattering rate $\Gamma_{\text {sc }}$ obtained from Eq. (11) is proportional to $\omega^{4}$, i.e., it has the same frequency dependence as Rayleigh scattering.

For the calculation of the SRS gain factor $G_{\Pi}$ and linewidth $\delta \bar{\nu}_{G}$ [using Eq. (6)] the sum of the frequencydependent contributions of the anharmonic decay [Eq. (10)] and the scattering of the polariton [Eq. (11)] replace the damping constant $\Gamma_{0}$ in the denominator of the dielectric function [Eq. (5)]. The calculated result for the linewidth $\delta \bar{\nu}_{G}$ in $\mathrm{ZnO}$-doped $\mathrm{LiNbO}_{3}$ at $77 \mathrm{~K}$ is shown in Fig. 4(b) as dotted curve. The steep decrease of $\delta \bar{\nu}_{G}$ below the optical phonon frequency is predicted correctly by the calculations and provides the background for the measured maxima and minima, which are caused by coupling to low-frequency excitations.

\section{Coupling to low-frequency modes}

The influence of low-frequency modes on the damping and the dispersion relation of polaritons has been discussed in the literature for $\mathrm{LiNbO}_{3}$ and $\mathrm{LiTaO}_{3}$. Two limiting cases of the coupling mechanisms are usually considered: First, the coupling of the low-frequency modes to the mechanical part of the polariton ${ }^{7,11}$ and second direct coupling to the light field, ${ }^{6}$ assuming that each mode has its own oscillator strength. In this paper we discuss in detail the first mechanism and apply it to our experimental results. We have found, however, that our experimental results can also be described by the second coupling mechanism. Our results are not sensitive enough to distinguish clearly between both mechanisms. 
We do not include in our treatment the coupling of the polaritons to a Debye relaxational mode, which has been shown to be important for polariton damping in $\mathrm{LiNbO}_{3}$ below $80 \mathrm{~cm}^{-1}$ at room temperature. ${ }^{10}$ At $77 \mathrm{~K}$ the relaxation mode is not effective.

Each of the $n$ low-frequency modes is described by a damped oscillator with resonance frequency $\omega_{b}$, damping constant $\Gamma_{b}$, and mass $m_{b}$, coupled to the mechanical part of the polariton by a symmetric coupling constant $K_{0 b}$ $=K_{b 0}(b=1$ to $n)$ :

$$
m_{b}\left(\omega_{b}^{2}-\omega^{2}-i \Gamma_{b} \omega\right) \widetilde{\mathbf{Q}}_{b}=K_{b 0} \widetilde{\mathbf{Q}},
$$

$$
m\left(\omega_{0}^{2}-\omega^{2}-i \Gamma_{\Pi}\left(\omega_{\Pi}\right) \omega\right) \widetilde{\mathbf{Q}}=e \widetilde{\mathbf{E}}+\sum_{b=1}^{n} K_{0 b} \widetilde{\mathbf{Q}}_{b},
$$

where $\Gamma_{\Pi}$ is the sum of the contributions of the anharmonic decay and the scattering at defects [Eqs. (10) and (11)].

With help of the Eq. (12a) $\mathbf{Q}_{b}$ is eliminated in Eq. (12b). Then this equation is solved for $\mathbf{Q}$ and used for the calculation of the dielectric function. This results in a dielectric function, in which the low-frequency modes occur in the denominator, increase the damping of the polaritons, and influence the dispersion relation. We get

$$
\varepsilon_{\Pi}(\omega)=\varepsilon_{\infty}+\frac{S_{0} \omega_{0}^{2}}{\omega_{0}^{2}-\omega^{2}-i \omega \Gamma_{\Pi}\left(\omega_{\Pi}\right)+\sum_{b=1}^{n}\left\{K_{0 b} K_{b 0} /\left[m_{b} m\left(\omega_{b}^{2}-\omega^{2}-i \Gamma_{b} \omega\right)\right]\right\}} .
$$

For this coupling mechanism of the low-frequency modes to the polariton, the real and imaginary part of the dielectric function obey the Kramers-Kronig relation. ${ }^{32}$ The dielectric function is the essential quantity both for stimulated and spontaneous Raman scattering. The results of the calculations [using Eq. (13)] will be compared with our experimental results on spontaneous and stimulated Raman scattering in the following.

We have investigated spontaneous Raman scattering from the $256-\mathrm{cm}^{-1} A_{1}$ mode of $\mathrm{LiNbO}_{3}$ crystals. The scattering angle $\vartheta$ was $90^{\circ}$, i.e., we investigated polaritons with a large wave vector $\left(k_{\Pi} \approx \sqrt{2} k_{P}\right)$ and with a frequency close to that of the optical phonon $\left(\omega_{\Pi} \approx \omega_{0}\right)$. In this case, the following simple equation is a good approximation for the spontaneous scattering cross section: ${ }^{16}$

$$
\frac{d^{2} \sigma}{d \Omega d \omega} \propto[n(\omega, T)+1] \operatorname{Im}\{\varepsilon(\omega)\} .
$$

In Fig. 5 the spontaneous Raman spectra of $\mathrm{ZnO}: \mathrm{LiNbO}_{3}$ and nearly stoichiometric $\mathrm{LiNbO}_{3}(\mathrm{Li}: \mathrm{Nb}=49.5: 50.5)$ at 77 $\mathrm{K}$ are presented on a normal scale and a scale enlarged by a factor 100 to show clearly the weak bands in the lowfrequency wing of the 256- $\mathrm{cm}^{-1}$ line. In $\mathrm{ZnO}: \mathrm{LiNbO}_{3}$ peaks at $112,150,163,190$, and $199 \mathrm{~cm}^{-1}$ are clearly seen. A careful quantitative analysis reveals additional weak peaks, which are listed in Table I. We calculated the spontaneous Raman intensity using Eqs. (13) and (14) and fitted the result to the measured spectrum to get frequencies $\bar{\nu}_{b}$, linewidths $\Gamma_{b}$, and coupling strengths $K_{0 b} K_{b 0} / m_{b} m$ of the lowfrequency modes. The analysis was also carried out for the nearly stoichiometric $\mathrm{LiNbO}_{3}$ crystal, and the results are given in Table I.

Next, we compare the calculations including the lowfrequency excitations with our gain measurements, in particular with the height $G_{\Pi}$ and the linewidth $\delta \bar{\nu}_{G}$ of the gain curve (see Figs. 3 and 4 ). The frequencies $\bar{\nu}_{b}$, linewidths $\Gamma_{b}$, and coupling strengths $K_{0 b} K_{b 0} / m_{b} m$ in Eq. (13) were taken from the spontaneous Raman-scattering experiments (see Table I). The result is not shown in the figures, because the calculated maxima of the linewidth were higher than the measured ones and the corresponding minima of the gain factor $G_{\Pi}$ were lower than the measured ones. Therefore, we reduced the coupling strengths by the factor $R_{b}$ given in Table I. Then good agreement is obtained between the calculated solid curves and the measured points in Figs. 3 and 4. It is not clear at present why the coupling strengths obtained from spontaneous Raman scattering have to be reduced to obtain agreement with the stimulated scattering results. It should be remembered, however, that in the spontaneous Raman measurements the wave vector $k_{\Pi}$ is large $(\approx 3.7$ $\left.\times 10^{5} \mathrm{~cm}^{-1}\right)$, while the gain measurements are carried out for small values of $k_{\Pi}\left(<2 \times 10^{4} \mathrm{~cm}^{-1}\right)$. A tentative explanation is that the coupling strengths depend slightly on the wave vector $k_{\Pi}$ of the polariton. This would be first evidence that the dielectric constant may depend on the wave vector in addition to its frequency dependence [Eq. (13)].

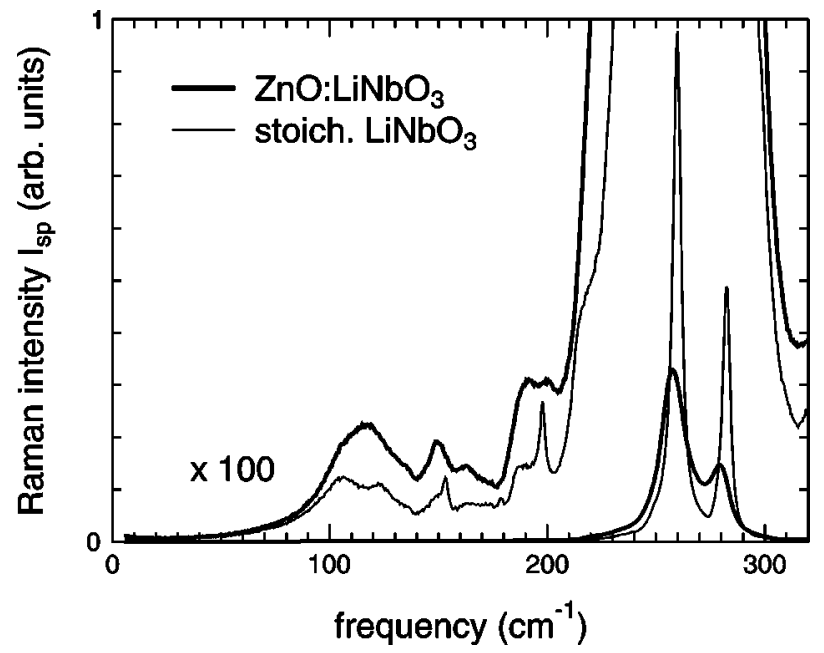

FIG. 5. Spontaneous Raman spectra in $A_{1}$ symmetry of $\mathrm{ZnO}: \mathrm{LiNbO}_{3}$ and nearly stoichiometric $\mathrm{LiNbO}_{3}$ at $T=77 \mathrm{~K}(\vartheta$ $\left.=90^{\circ}, k_{\Pi} \approx \sqrt{2} k_{P}\right)$. Both spectra are scaled to identical oscillator strength of the optical phonon at $\bar{\nu}_{0}=256 \mathrm{~cm}^{-1}$ (spectral resolution: $\left.1.3 \mathrm{~cm}^{-1}\right)$. 
TABLE I. Low-frequency modes obtained from spontaneous Raman scattering: frequency $\bar{\nu}_{b}$, linewidth $\Gamma_{b}$, coupling strength $K_{0 b} K_{b 0} / m_{b} m . R_{b}$ is the ratio of the coupling strengths used in the spontaneous and stimulated Raman experiments.

\begin{tabular}{lcccll}
\hline \hline & \multicolumn{5}{c}{$K_{0 b} K_{b 0} /$} \\
Crystal & $\bar{\nu}_{b}$ & $\Gamma_{b}$ & $\begin{array}{c}m_{b} m \\
\left(\mathrm{~cm}^{-1}\right)\end{array}$ & & Suggested \\
$\left(\mathrm{cm}^{-1}\right)$ & $\left(10^{6} \mathrm{~cm}^{-4}\right)$ & $R_{b}$ & cause \\
\hline ZnO:LiNbO $_{3}$ & 112 & 33 & 12.0 & 0.5 & Defect mode \\
& 122 & 20 & 3.5 & 0.5 & Defect mode \\
& 134 & 12 & 1.0 & 0.5 & Defect mode \\
& 150 & 12 & 2.7 & 0.5 & $E$ mode(TO) \\
& 163 & 14 & 2.0 & 0.6 & Defect mode \\
& 190 & 12 & 3.0 & 0.5 & Defect mode \\
& 199 & 11 & 1.3 & 0.6 & $E$ mode(LO) \\
Nearly & 222 & 14 & 4.0 & 0.8 & Defect mode \\
stoich. & 235 & 14 & 4.0 & 0.8 & $E$ mode(TO) \\
LiNbO 3 & 106 & 33 & 9 & 0.7 & Defect mode \\
& 125 & 20 & 3 & 0.3 & Defect mode \\
& 148 & 10 & 0.8 & 0.5 & Defect mode \\
& 153 & 8.0 & 1.1 & 0 & $E$ mode(TO) \\
& 167 & 20 & 1.2 & 1.1 & Defect mode \\
& 188 & 13 & 1.7 & 0.3 & Defect mode \\
& 198 & 3 & 2.0 & 0 & $E$ mode(LO) \\
& 217 & 10 & 2.0 & 0.5 & Defect mode \\
& 233 & 10 & 1.8 & 0.7 & Defect mode \\
& 239 & 4 & 0.7 & 0.7 & $E$ mode(TO) \\
\hline \hline & & & & & \\
& & & &
\end{tabular}

The comparison between the experimental results and the calculations of the gain factor $G_{\Pi}$ and the linewidth $\delta \bar{\nu}_{G}$ showed that the frequency dependence of the polariton damping caused by anharmonic decay and elastic scattering is important. However, the distinct maxima of the polariton damping are due to their coupling to low-frequency modes.

We have also determined the dispersion relation of the polaritons, which is defined in the literature in different ways. ${ }^{14,16}$ In our case, the dispersion curve was obtained from the experiments and calculations by determining the frequency $\bar{\nu}_{\Pi}$ of the maximum $G_{\Pi}$ of the gain curve and plotting it versus the corresponding polariton wave vector $k_{\Pi}$, which was calculated from the phase matching angle $\vartheta$ [Eq. (2)]. It should be mentioned that for constant damping $\Gamma_{0}$ [see Eq. (5)] this dispersion curve agrees in a very good approximation with that of an undamped polariton $\left(\Gamma_{0}\right.$ $=0)$. Because of the high precision of the measurements it was possible to observe the influence of the low-frequency modes on the dispersion curve.

We determined the difference $\Delta \bar{\nu}_{\Pi}$ between the polariton frequencies with and without low-frequency modes and plotted $\Delta \bar{\nu}_{\Pi}$ versus polariton frequency $\bar{\nu}_{\Pi}$ (see Fig. 6). The solid curve represents the calculations including the lowfrequency modes using Eqs. (6) and (13). There is good agreement between the experimental points and the solid curve. The modulation of the frequency difference $\Delta \bar{\nu}_{\Pi}$ is caused by the low-frequency modes, the frequencies of which are indicated by the short vertical lines. $\Delta \bar{\nu}_{\Pi}$ is lowered below the resonance frequency of each mode and lifted above this frequency, indicating that larger coupling strengths would cause a splitting of the dispersion curve. But in $\mathrm{ZnO}: \mathrm{LiNbO}_{3}$ no splitting was observed, in contrast to congruent $\mathrm{LiNbO}_{3}$, where fs transient grating experiments showed a splitting of the dispersion curve. ${ }^{6}$

\section{INTERPRETATION OF THE LOW-FREQUENCY MODES}

In this section we discuss the physical origin of the lowfrequency modes observed in the amplifier and spontaneous scattering measurements (Figs. 3-5). The frequencies of the modes at 150 (153), 199 (198), and 235 (239) $\mathrm{cm}^{-1}$ in $\mathrm{ZnO}$-doped (nearly stoichiometric) $\mathrm{LiNbO}_{3}$ (see Table I) are close to those of the $E$ modes at 152 (TO), 194 (LO), and $236 \mathrm{~cm}^{-1}$ (TO). ${ }^{33}$ There exist two reasons for observing these modes in the $A_{1}$ mode spectrum: First, the $E$ modes can couple to the $A_{1}$ modes due to internal strain in the crystal. ${ }^{7,23}$ Second, the $E$ modes, which are polarized perpendicular to the $A_{1}$ modes, are not completely suppressed because of leakage of the polarizers in the experiments. The sharp peaks at 153 and $198 \mathrm{~cm}^{-1}$ in nearly stoichiometric $\mathrm{LiNbO}_{3}$ in Fig. 5 are certainly caused by the second effect.

Next we discuss the prominent modes in the frequency range from 100 to $130 \mathrm{~cm}^{-1}$. In previous papers ${ }^{23,34}$ two low-frequency modes around $100 \mathrm{~cm}^{-1}$ have been observed in spontaneous Raman scattering and attributed to missing $E$ modes of $\mathrm{LiNbO}_{3}$. But Yang et al. ${ }^{33}$ concluded from the directional dispersion of all zone-center modes, that the number of $E$ modes is complete, because the lowest $E$ mode at $152 \mathrm{~cm}^{-1}$ is doubly degenerate and there is an additional $E$ mode at $530 \mathrm{~cm}^{-1}$. The spontaneous Raman spectra in Fig. 5 show that the maxima at 106 and $120 \mathrm{~cm}^{-1}$ are clearly separated in nearly stoichiometric $\mathrm{LiNbO}_{3}$, while a broad band with a weak shoulder is observed in this frequency region for $\mathrm{ZnO}: \mathrm{LiNbO}_{3}$. The coupling strengths for both modes are lower in the nearly stoichiometric crystal than in the doped congruent crystal (see Table I). In a further better stoichiometric crystal $(\mathrm{Li}: \mathrm{Nb}=50.0: 50.0$ with an accuracy of \pm 0.1$)$ these Raman bands were not observed within the experimental accuracy. We conclude from these results that the modes at about 106 and $120 \mathrm{~cm}^{-1}$ are defect modes, which depend on the stoichiometry and doping of the $\mathrm{LiNbO}_{3}$ crystals.

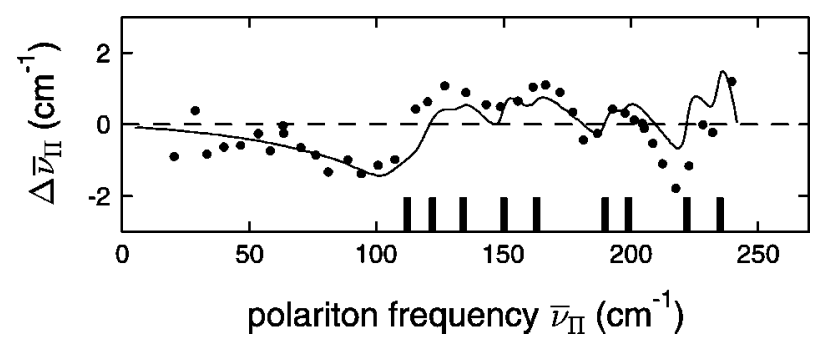

FIG. 6. Difference $\Delta \bar{\nu}_{\Pi}$ between the polariton frequencies with and without low-frequency modes. The full circles represent the experimental points. The solid curve has been calculated taking into account the low-frequency modes. The short vertical lines mark the resonance frequencies of the low-frequency modes. 
It is difficult to draw conclusions on the physical origin of the other low-frequency modes in Table I, because there are only minor differences in the spontaneous Raman spectra (Fig. 5) and in the gain factor and linewidth (Figs. 3 and 4) of $\mathrm{ZnO}$-doped and nearly stoichiometric $\mathrm{LiNbO}_{3}$. However, some of the modes have different frequencies in both crystals, and for those modes having approximately the same frequency the coupling strength is lower in the stoichiometric crystal. These results indicate that these modes may also be related to defect centers.

We treated the low-frequency modes in Eq. (12a) as damped harmonic oscillators, which is a good model e.g. for localized vibrational modes of the defect centers. However, there may be a coupling of the polaritons to shallow traps, which are known in $\mathrm{LiNbO}_{3}$ in the context of the dynamics of the photorefractive effect and the dark traces. ${ }^{30}$ The activation energies of these traps are comparable with the observed energies of the low-frequency modes (in the range from 90 to $120 \mathrm{~cm}^{-1}$ ) and vary with crystal composition, dopant and doping concentration. ${ }^{35}$

A completely different model for the explanation of the low-frequency excitations in congruent $\mathrm{LiNbO}_{3}$ has been introduced by Bakker et al. ${ }^{6}$ They discuss their results in terms of transitions between higher energy levels of a strongly anharmonic triple-well potential. However, we think that this model is not able to explain our experimental results for two reasons. First, the maxima and minima of the gain factor and the linewidth are observed even at $20 \mathrm{~K}$, where the higher levels of the anharmonic potential are not populated thermally. Second, we found pronounced differences between the doped congruent and the undoped nearly stoichiometric crystals. It is difficult to imagine corresponding changes of the anharmonic potential with stoichiometry or doping of the crystals.

\section{CONCLUSIONS}

The damping of phonon polaritons of the $256-\mathrm{cm}^{-1} A_{1}$ mode of $\mathrm{LiNbO}_{3}$ has been investigated by measuring the gain factor and linewidth of stimulated scattering from polaritons and the spontaneous Raman spectra of the optical phonon. The theoretical treatment of the gain factor for stimulated scattering from polaritons is based on the solution of the coupled wave equations for the Stokes and polariton fields and the oscillator equation for the mechanical part of the polariton. The gain factor was shown to be related to the dielectric function of the crystal, which contains the damping of the polariton. We measured the gain factor and the linewidth in an amplifier experiment for polariton frequencies from 30 to $230 \mathrm{~cm}^{-1}$ in $\mathrm{ZnO}$-doped congruent $\mathrm{LiNbO}_{3}$ and undoped nearly stoichiometric $\mathrm{LiNbO}_{3}$ at $77 \mathrm{~K}$. Spontaneous Raman spectra were recorded for a scattering angle of $90^{\circ}$, i.e., for the optical phonon at $256 \mathrm{~cm}^{-1}$, with special emphasis upon the wing of the Raman line. A careful analysis of the experimental data and a comparison with the theory gave the following information on the damping of the polaritons. The damping by the anharmonic decay of the phonon part of the polariton into two acoustic phonons and the scattering of the polaritons at crystal defects decrease strongly with polariton frequency and provide a measurable contribution only below the optical-phonon frequency (about 200-256 $\mathrm{cm}^{-1}$ ). The main damping mechanism in the investigated polariton frequency range is the coupling of a large number of lowfrequency excitations to the mechanical or electromagnetic part of the polariton. They cause pronounced peaks in the frequency dependence of the polariton damping.

We have tried to clarify the physical origin of the lowfrequency excitations. Three of these excitations have been identified as $E$ modes, which can couple to the $256-\mathrm{cm}^{-1} A_{1}$ mode by internal strain. Two modes, in the range from 100 to $130 \mathrm{~cm}^{-1}$, have been shown to be connected with defects in the $\mathrm{ZnO}$-doped congruent or undoped nearly stoichiometric $\mathrm{LiNbO}_{3}$ crystals. The rest of the modes is suggested to be related to defects of unknown nature in the undoped stoichiometric and doped congruent crystals. For an identification of the defects investigations combining different physical methods, like ESR, photoconductivity, thermoluminescence, with the methods discussed in this paper, are necessary.

\section{ACKNOWLEDGMENTS}

The authors would like to thank O. F. Schirmer for the nearly stoichiometric crystal, Tiequn Qiu for many helpful discussions, and T. Tyroller for the spontaneous Raman spectra. This work was supported by the Deutsche Forschungsgemeinschaft.
*Present Address: Ulrich T. Schwarz, C15 Clark Hall, Cornell University, Ithaca, NY 14853-2501.

${ }^{1}$ D. H. Auston and M. C. Nuss, IEEE J. Quantum Electron. 24, 184 (1988).

${ }^{2}$ T. P. Dougherty, G. P. Wiederrecht, and K. A. Nelson, J. Opt. Soc. Am. B 9, 2179 (1992).

${ }^{3}$ P. C. M. Planken, L. D. Noordam, J. T. M. Kennis, and A. Lagendijk, Phys. Rev. B 45, 7106 (1992).

${ }^{4}$ H. J. Bakker, S. Hunsche, and H. Kurz, Phys. Rev. Lett. 69, 2823 (1992)

${ }^{5}$ D. P. Kien, J. C. Loulergue, and J. Etchepare, Opt. Commun. 101, 53 (1993)

${ }^{6}$ H. J. Bakker, S. Hunsche, and H. Kurz, Phys. Rev. B 50, 914 (1994); Rev. Mod. Phys. 70, 523 (1998).

${ }^{7}$ G. P. Wiederrecht, T. P. Dougherty, L. Dhar, K. A. Nelson, D. E.
Leaird, and A. M. Weiner, Phys. Rev. B 51, 916 (1995).

${ }^{8}$ G. M. Gale, F. Vallée, and C. Flytzanis, Phys. Rev. Lett. 57, 1867 (1986).

${ }^{9}$ F. Vallée and C. Flytzanis, Phys. Rev. B 46, 13799 (1992).

${ }^{10}$ T. Qiu and M. Maier, Phys. Rev. B 56, R5717 (1997).

${ }^{11}$ U. T. Schwarz and M. Maier, Phys. Rev. B 53, 5074 (1996).

${ }^{12}$ S. Ushioda and J. D. McMullen, Solid State Commun. 11, 299 (1972); S. Ushioda, J. D. McMullen, and M. J. Delaney, Phys. Rev. B 8, 4634 (1973).

${ }^{13}$ A. F. Penna, A. Chaves, P. da R. Andrade, and S. P. S. Porto, Phys. Rev. B 13, 4907 (1976).

${ }^{14}$ R. Claus, L. Merten, and J. Brandmüller, Light Scattering by Phonon Polaritons, Springer Tracts in Modern Physics Vol. 75 (Springer, Berlin, 1975), Chap. 4.10.

${ }^{15}$ H. J. Bakker, S. Hunsche, and H. Kurz, Phys. Rev. B 48, 13524 (1993). 
${ }^{16}$ A. S. Barker, Jr., and R. Loudon, Rev. Mod. Phys. 44, 18 (1972).

${ }^{17}$ U. T. Schwarz, Ph. D. thesis, Universität Regensburg, 1997.

${ }^{18}$ H. Amler, S. Pichlmaier, P. Ramm, and M. Maier, Opt. Commun. 97, 397 (1993).

${ }^{19}$ C. H. Henry and C. G. B. Garrett, Phys. Rev. 171, 1058 (1968).

${ }^{20}$ S. S. Sussman, Microwave Laboratory, W. W. Hansen Laboratories of Physics, Stanford University, Stanford, California, Report No. 1851, 1970 (unpublished).

${ }^{21}$ M. Maier, Appl. Phys. Lett. 11, 209 (1976).

${ }^{22}$ V. G. Dmitriev, G. G. Gurzadyan, and D. N. Nikogosyan, Handbook of Nonlinear Optical Crystals (Springer, Berlin, 1991), p. 74.

${ }^{23}$ W. D. Johnston, Jr. and I. P. Kaminow, Phys. Rev. 168, 1045 (1968).

${ }^{24}$ U. T. Schwarz and M. Maier, Phys. Rev. B 55, 11041 (1997).

${ }^{25}$ K. Okubo and S.-I. Tamura, Phys. Rev. B 28, 4847 (1983).

${ }^{26}$ M. R. Chowdhury, G. E. Peckham, and D. H. Saunderson, J. Phys. C 11, 1671 (1978).
${ }^{27}$ S. K. Bose, S. Kirkpatrick, and W. M. Dennis, J. Lumin. 72-74, 422 (1997).

${ }^{28}$ S. Kojima, Jpn. J. Appl. Phys., Part 1 32, 4373 (1993).

${ }^{29}$ G. I. Malovichko et al., Appl. Phys. A: Solids Surf. 56, 103 (1993).

${ }^{30}$ O. F. Schirmer, H.-J. Reyher, and M. Wöhlecke, in Insulating Materials for Optoelectronics, edited by T. Aquilló-López (World Scientific, Singapore, 1995), Chap. 4.

${ }^{31}$ G. P. Srivastava, The Physics of Phonons (Adam Hilger, Bristol, 1990), p. 178.

${ }^{32}$ A. S. Barker, Jr., Phys. Rev. 165, 917 (1968).

${ }^{33}$ X. Yang, G. Lan, B. Li, and H. Wang, Phys. Status Solidi B 142, 287 (1987).

${ }^{34}$ A. M. Bel'skii, I. M. Gulis, and K. A. Saechnikov, Quantum Electron. 25, 810 (1995).

${ }^{35}$ K. L. Sweeney, L. E. Halliburton, D. A. Bryan, R. R. Rice, R. Gerson, and H. E. Tomaschke, J. Appl. Phys. 57, 1036 (1985). 